\title{
Addition of exogenous polypeptides on the mammalian reovirus outer capsid using reverse genetics
}

Virginie Brochu-Lafontaine, Guy Lemay*

Département de microbiologie et immunologie, Université de Montréal, Montréal, Qc.

Canada $\mathrm{H} 3 \mathrm{C} 3 \mathrm{J7}$

${ }^{*}$ Corresponding author. Tel.: 514 3432422; fax: 5143435701.

E-mail address: guy.lemay@umontreal.ca (G. Lemay) 


\begin{abstract}
Addition of exogenous peptide sequences on viral capsids is a powerful approach to study the process of viral infection or to retarget viruses toward defined cell types. Until recently, it was not possible to manipulate the genome of mammalian reovirus and this was an obstacle to the addition of exogenous sequence tags onto the capsid of a replicating virus. This obstacle has now been overcome by the advent of the plasmid-based reverse genetics system. In the present study, reverse genetics was used to introduce different exogenous peptides, up to 40 amino acids long, at the carboxyl-terminal end of the $\sigma 1$ outer capsid protein. The tagged viruses obtained were infectious, produce plaques of similar size, and could be easily propagated at hight titers. However, attempts to introduce a 750 nucleotides-long sequence failed, even when it was added after the stop codon, suggesting a possible size limitation at the nucleic acid level.
\end{abstract}

Keywords: Reovirus; Reverse genetics; Tag; Epitope 


\section{Introduction}

Mammalian reovirus under the trademark "Reolysin ${ }^{\circledR}$ " (derived from reovirus serotype 3 Dearing: T3/Human/Ohio/Dearing/55) is presently under study as an "oncolytic" virus in various clinical assays for the treatment of human cancers (recently reviewed in: (Kelly et al., 2009; Lal et al., 2009; Thirukkumaran and Morris, 2009; Harrington et al., 2010). Reovirus, is among the few viruses that can be used as an oncolytic agent without prior genetic engineering. Reovirus is only weakly pathogenic in human adults and its replication is blocked in "normal" cells while it is able to replicate in, propagate, and destroy transformed/cancer cells; this is apparently due to the presence of an activated Ras oncogenic pathway in these cells. Direct or indirect activation of the Ras signalling pathway appears to increase viral uncoating, repress innate immunity and/or augment viral-induced apoptosis, resulting in the natural oncolytic activity of the virus (Alain et al., 2007; Marcato et al., 2007; Rudd and Lemay, 2005; Shmulevitz et al., 2010; Smakman et al., 2005; Strong et al., 1998).

The reovirus capsid is made of two concentric layers of proteins; the trimeric $\sigma 1$ protein is exposed at the surface of the virion and binds onto target cells; the carboxylterminal globular domain being most exposed and responsible for high-affinity binding to the main cellular receptor, JAM-A. Reovirus virions enter cells by endocytosis and are partially uncoated before being released to the cytoplasm; in contrast, extracellular proteases can partially uncoat the virions to infectious subviral particles (ISVPs) that still harbor $\sigma 1$ at their surface but that directly cross the plasma membrane to enter the cytoplasm where viral replication ensues (Danthi et al., 2010).

Despite its natural oncolytic ability, it is likely that reovirus binding onto normal cells via ubiquitous receptors such as sialic acid, JAM-A, and other unidentified receptor(s) (Antar et al., 2009; Coyne, 2009) will limit efficiency of the virus to reach some target cells in the 
organism. There are also some evidence to suggest that the virus receptors on the cell surface are either absent, or "masked" in some in some human cancer cell types, such as colorectal cancer cells (van Houdt et al., 2008). Finally, even though the virus appears to be only weakly pathogenic in humans, it cannot be excluded totally that some individuals, especially younger or immunodeficient patients, could develop disease due to reovirus inoculation, especially after systemic (intravenous) administration. Although its involvement in human biliary atresia of the newborn remains to be proven more conclusively, a significantly higher prevalence of reovirus RNA was detected in hepatic tissues from diseased individuals compared to normal controls (Tyler et al., 1998). Given strains of reovirus were also shown to induce liver pathologies, that are reminiscent of biliary atresia, in a murine experimental model (Parashar, Tarlow, and McCrae, 1992; Forrest and Dermody, 2003). The virus was also shown to induce "black foot syndrome" in immunodeficient SCID/NOD mice (Loken et al., 2004). There are thus possibly important benefits in the development of reovirus strains that are either attenuated (Kim et al., 2011) or targeted to bind specifically onto given cancer cell types, as proposed by others (Van Den Wollenberg et al., 2009). This last approach is currently under study for various oncolytic viruses such as adenovirus, measles virus, and herpesvirus, among others (Blechacz and Russell, 2008; Mathis, Stoff-Khalili, and Curiel, 2005; CampadelliFiume et al., 2011).

Until recently, it was not possible to modify the genome of reovirus, using the socalled "reverse genetics" approach that has been so widely used to study viruses belonging to most virus families. Nevertheless, a small 6-amino acids long peptide was added to the extreme carboxyl-terminal end of $\sigma 1$ using a complex experimental approach in which cells are transfected with the plasmid encoding the tagged protein and then infected with a wild type virus to select recombinant viruses (van den Wollenberg et al., 2008). This strategy appears quite complex and requires an adequate cell line for the selection procedure. 
A plasmid-based reverse genetics system was more recently described for mammalian reovirus by the group of Dr Terence Dermody (Kobayashi et al., 2007; reviewed by: Lemay, 2011); this novel approach was used by this group to study various reovirus mutants obtained by site-directed mutagenesis (see for example: (Boehme, Guglielmi, and Dermody, 2009; Danthi et al., 2008a; Danthi et al., 2008b; Kobayashi et al., 2007; Kobayashi et al., 2009)), or to substitute a whole gene segment from one reovirus serotype to the other (Kobayashi et al., 2007; Zurney et al., 2009).

In the present study, the usefulness of the plasmid-based reverse genetics approach was further established by adding different epitope tags at the carboxyl-terminal end of $\sigma 1$. Viruses harbouring up to a 40 amino acids long peptide were readily recovered. However, infectious viruses could not be recovered when the sequence encoding the yellow fluorescent protein was fused to $\sigma 1$, even when a stop codon was introduced to avoid the production of a fusion protein. This suggests the possibly of a size limitation of exogenous sequences that can be added at the nucleic acid level, independently of protein structure or function. 


\section{Materials and methods}

\subsection{Cells and viruses}

L929 cells and Vero cells were originally obtained from the American Type Culture Collection and were used for reovirus propagation and titration; BHK21 cells stably expressing the T7 RNA polymerase (Buchholz, Finke, and Conzelmann, 1999) were a generous gift from the laboratory of Dr John Hiscott (Lady Davis Research Institute, Montréal, Canada). All these cells were grown in minimal Eagle medium (MEM; Wisent) containing 5\% fetal bovine serum (FBS Gold, PAA laboratories).

\subsection{Antibodies}

Hybridoma cell lines producing either anti- $\sigma 3(4 \mathrm{~F} 2)$ or anti- $\mu 1(10 \mathrm{~F} 6)$ have been described before (Virgin et al., 1991) and were obtained from Dr Kevin Coombs (Department of Microbiology, Manitoba University). These cells were grown in MEM for suspension culture with $10 \%$ fetal bovine serum, proline $(20 \mu \mathrm{g} / \mathrm{ml})$ and $\beta$-mercaptoethanol $(50 \mu \mathrm{M})$; they were fed each 2 or 3 days to obtain cell concentration between $2 \times 10^{5}$ and $5 \times 10^{5}$ per ml, they were resuspended in fresh medium at a concentration of $5 \times 10^{5}$ cells per ml and left until most cells were dead as judged by trypan blue exclusion; cells and debris were removed by centrifugation at low speed and supernatants containing the monoclonal antibodies were kept at $4{ }^{\circ} \mathrm{C}$ with $1 \mathrm{mM}$ sodium azide as preservative. The polyclonal antiserum directed against the carboxyl-terminal head domain of $\sigma 1$ was produced originally in the laboratory of Dr Terence Dermody (Vanderbilt University, Tennessee) and was a generous gift from Dr Earl Brown (University of Ottawa). The mouse anti-polyhistidine monoclonal antibody was obtained from Qiagen (Qiagen, Mississauga, Ont., mouse Penta-His antibody, Catalog \#34660). The mouse anti-HA tag (12CA5) was from Berkeley Antibody Company (BabCO) and a generous gift 
from the laboratory of Dr Pierre Belhumeur (Département de microbiologie et immunologie, Université de Montréal). The rabbit anti-tubulin was obtained from ICN Biomedicals Inc.

\subsection{Plasmid constructs}

The plasmids harbouring separately each of the cDNA corresponding to the 10 genes of reovirus serotype 3 Dearing, under the transcriptional control of the $\mathrm{T} 7$ promoter, have been described (Kobayashi et al., 2007) and were obtained from the laboratory of Dr Terence Dermody (Vanderbilt University, Nashville, Tennessee). Medium scale plasmid DNA was column-purified using Qiagen plasmid midi kit and endotoxin-free buffers (Catalog \#12243 and \#19048), as recommended by the manufacturer. Concentration and purity were determined by measuring optical density using a nanodrop microspectrophotometer (ND1000, Thermo Scientific). To insert sequence encoding for the hexahistidine or single HA tag at the end of the $\sigma 1$-encoding sequence, the corresponding plasmid was submitted to sitedirected mutagenesis using the QuickChange ${ }^{\circledR}$ Lightning site-directed mutagenesis kit (Stratagene, Catalog \#200518), as recommended by the manufacturer. In an additional construct, a unique SpeI site was introduced following the hexahistidine tag, just before the stop codon, thus adding two amino acids to the protein (a threonine and a serine); this was also done by site-directed mutagenesis using the plasmid encoding hexahistidine-tagged $\sigma 1$. This same plasmid was used to introduce 3 additional copies of the HA tag. The pMPY-3xHA plasmid (Schneider et al., 1995) was used as a template for PCR amplification of the 3HAencoding region using oligonucleotides designed to introduce the SpeI site at both ends of the PCR product that was cloned thereafter at the SpeI site following the hexahistidine tag. The sequence encoding the enhanced yellow fluorescent protein was amplified from the pcDNA.1.eYFP.MCS(MB) plasmid (a generous gift from Dr Jacques Thibodeau) and also subcloned at the SpeI site. In a final construct, a stop codon was introduced by site-directed 
mutagenesis 3 amino acids after the beginning of the YFP-encoding sequence. The presence of the expected mutations or insertions, and absence of other changes in the adjacent region,was verified by sequencing the final plasmids on small-scale preparation of endotoxinfree plasmid DNA that was column-purified, as recommended by the manufacturer (Zyppy plasmid miniprep kit, Zymo Research). The different oligonucleotides used as primers for mutagenesis, PCR and sequencing are presented in Table I.

\subsection{Recovery of infectious viruses by plasmid-based reverse genetics}

Recovery of infectious reovirus stocks was done using a modification of the original procedure (Kobayashi et al., 2007). The nine wild-type plasmids, prepared as medium scale preparation, were simultaneously introduced with the plasmid encoding the tagged $\sigma 1$; transfection was done using Fugene 6 (Roche), as recommended by the manufacturer. Approximately $0.10 \mu \mathrm{g}$ of each plasmid was used to transfect semi-confluent $35 \mathrm{~mm}$-diameter petri dish of BHK21 cells that express the T7 RNA polymerase. The medium was recovered 3-4 days later, cells were trypsinized and plated in a $100 \mathrm{~mm}$-diameter petri dish with the medium recovered previously and completed with $9 \mathrm{ml}$ of complete medium containing $5 \%$ heat-inactivated fetal bovine serum. Two-third of the medium was replaced with fresh medium, 3 days later, and incubated for another 4 days before being frozen at $-80{ }^{\circ} \mathrm{C}$ and used as starting virus stocks. Virus clones were recovered from single plaques obtained on Vero cells in the presence of chymotrypsin; plaques were visualized directly by phase contrast microscopy without staining. The clones were amplified on Vero cells in the presence of chymotrypsin, titrated, and further propagated on L929 cells at an approximate MOI of one PFU/cell before being titrated again. 


\subsection{Plaque assay}

Plaque assay was done using a modification of standard procedures. Briefly, Vero cells were plated in 6-wells plates, 24 before infection, in order to obtain confluent monolayers at the time of infection. After medium removal and washing with serum-free medium, serial tenfold dilutions of the virus samples were applied in minimal volume $(250 \mu l)$ in each well. Cells were left at $4{ }^{\circ} \mathrm{C}$ for one hour with gentle agitation each 15 minutes. Plates were briefly warmed at $37^{\circ} \mathrm{C} ; 2.5 \mathrm{ml}$ of $1 \%$ agar (bacto-agar, Difco) in serum-free medium 199 (prepared by mixing a $2 \%$ sterile agar solution with medium prepared at twice the normal concentration) was added to each well. The sterilized $2 \%$ agar solution was melted before in a microwave oven and kept at $45^{\circ} \mathrm{C}$ and mixed with the medium that was incubated at the same temperature before addition of $10 \mu \mathrm{g} / \mathrm{ml}$ of chymotrypsin (Sigma Sigma Type I-S from bovine pancreas; prepared at $10 \mathrm{mg} / \mathrm{ml}$ in $1 \mathrm{mM} \mathrm{HCl}$ and kept frozen in aliquots). After the agar overlay has hardened (a few minutes at room temperature), plates were incubated for different periods of time ( 4 to 5 days in routine titration assay). The cells were fixed by adding $2 \mathrm{ml}$ of $10 \%$ formaldehyde (in PBS) to each of the wells. After one hour at room temperature, the formaldehyde solution and the agar overlay were removed before adding $1 \mathrm{ml}$ of $1 \%$ crystal violet (in 70\% methanol). After 60 minutes, the staining solution was removed and the plates were washed in tap water before being air-dried. Plaque formation for recovery of infectious virus was performed similarly except that the plaques were identified directly by phase contrast microscopy using an inverted microscope. Individual plaques were picked with $1 \mathrm{ml}$ sterile pipette tips, transferred in $1 \mathrm{ml}$ of sterile medium and virus left to diffuse for at least 24 hours at $4^{\circ} \mathrm{C}$.

\subsection{Sequencing of S1 viral genome segment}

To verify that the sequences added were actually conserved in the final virus stocks obtained 
by reverse genetics, sequencing was done by RT-PCR amplification on the viral genome followed by direct sequencing of the PCR product. Virus stocks were used to infect L929 cells in $10 \mathrm{~cm}$-diameter petri dishes at a MOI of $1 \mathrm{PFU} / \mathrm{cell}$. Cells and medium were recovered at 24-30 hours post-infection, at which time most cells were lysed. Following two cycles of freeze-thaw $\left(-80^{\circ} \mathrm{C}\right.$ to room temperature), the lysate was extracted once with one-fourth volume of freon (1,1,2-Trichloro-1,2,2,-trifluoroethane, Mallinckrodt Chemicals). After 15 min of centrifugation at 7,500 rpm at $4{ }^{\circ} \mathrm{C}$ in a Sorvall SS34 rotor (in Corex $15 \mathrm{ml}$ tubes), supernatant was recovered and overlayed on a $1 \mathrm{ml}$ cesium chloride cushion at a density of $1.3 \mathrm{~g} / \mathrm{ml}$ for ultracentrifugation in a $70.1 \mathrm{Ti}$ rotor at $50,000 \mathrm{rpm}$ for one hour at $4{ }^{\circ} \mathrm{C}$. Virus pellet was recovered in $400 \mu \mathrm{l}$ of TEN buffer (10mM Tris-HCl pH 7.5, 1mM EDTA, 250mM $\mathrm{NaCl}$ ) before addition of $0.25 \%$ SDS followed by phenol-chloroform extraction and ethanol precipitation of viral double-stranded RNA. The RNA was denatured at $95^{\circ} \mathrm{C}$ for 5 min and cooled rapidly on ice. Reverse transcription on both strand of the double-stranded RNA was done with MLV reverse transcriptase, as recommended by the manufacturer (Roche) using one-fourth of the RNA and oligonucleotides specific for each strand at the ends of the gene

(Table I). One-fifth of the reverse transcriptase reaction was then used in a PCR reaction with the same two oligonucleotides for 35 cycles using the FastStart protocol (Roche). The PCR fragment was then purified using Qiaquick PCR purification kit (Qiagen) and sequenced by automated DNA sequencing (Applied Biosystems 3730 DNA Analyzer) in the sequencing service of Institut de Recherche en Immunologie et Cancérologie (IRIC) of Université de Montréal.

\subsection{Immunoblotting}

Infected cells were recovered by scraping in $500 \mu l$ of medium and centrifuged in an Eppendorf tube at $1000 \mathrm{rpm}$ for 30 seconds at $4{ }^{\circ} \mathrm{C}$. Cell pellets corresponding to a $60 \mathrm{~mm}-$ 
diameter petri dish were resuspended in $100 \mu \mathrm{l}$ of Tris- $\mathrm{HCl} 10 \mathrm{mM} \mathrm{pH} \mathrm{7.5,} \mathrm{1mM} \mathrm{EDTA,}$ $150 \mathrm{mM} \mathrm{NaCl}, 1 \%$ Nonidet P-40 and left on ice for 10 minutes before centrifugation at maximum speed for 5 minutes in an Eppendorf centrifuge at $4{ }^{\circ} \mathrm{C}$. Proteins were analyzed by SDS-PAGE and immunoblotting. Nitrocellulose membrane was blocked with $2 \%$ non-fat dry milk (Biorad) in TBS (Tris- $\mathrm{HCl} 10 \mathrm{mM} \mathrm{pH} \mathrm{7.5,150mM} \mathrm{NaCl)} \mathrm{and} \mathrm{sequentially} \mathrm{incubated} \mathrm{for}$ one hour at room temperature with the primary antibody. When using monoclonal antibodies from tissue culture medium, these were diluted with an equal volume of TBS containing the blocking agent and directly used. The diluted antibody solution was recovered and kept at $4{ }^{\circ} \mathrm{C}$ with $1 \mathrm{mM}$ sodium azide. Other primary antibodies were diluted in PBS containing $1 \%$ bovine serum albumin and sodium azide at either a 1/500 dilution for the anti- $\sigma 1$ antibody or at the dilution recommended by the manufacturer for the other antibodies. Membranes were washed in TBS containing 1\% Tween-20. Revelation was done using peroxydase-conjugated secondary antibody and chemiluminescent substrate as recommended by the manufacturer (Pierce SuperSignal West Dura Extended Duration Substrate). Images were obtained on Kodak BioMax Light Film.

\section{Results}

\subsection{Rationale}

As a proof of principle for the utilisation of the plasmid-based reverse genetics approach for addition of exogenous epitope to the reovirus outer capsid, two different small epitopes were first examined for their ability to be added at the carboxyl-terminal end of $\sigma$. This position was chosen since, in one study, an hexahistidine tag was already reported to be functional at this position (van den Wollenberg et al., 2008). Also, when a chimeric protein between the 
adenovirus fiber protein and $\sigma 1$ is added to adenovirus virions, the presence of both hexahistidine and myc tag at its carboxyl-terminal end still allowed cell binding by the $\sigma 1$ moiety (Mercier et al., 2004; Schagen et al., 2006; Tsuruta et al., 2005; 2007). In the present study, the hexahistidine epitope $(6 \mathrm{H})$ was first used and, as a further proof that different epitopes can be accommodated at this position, the more complex HA epitope, YPYDVPDYA (Kolodziej and Young, 1991; Wilson et al., 1984), was examined in parallel (Fig. 1A).

Analysis of the $\sigma 1$ crystallographic structure (Chappell et al., 2002)(PDB 1KKE) revealed that the peptide epitope should be presented at the external surface of each molecule of the trimeric structure (Fig. 1B), at the basis of the globular structure involved in binding to cellular protein JAM-A, acting as the main receptor for the virus (Fig 1C) (reviewed in: (Schelling et al., 2008)). However, the epitope will be located away from the known region of interaction between $\sigma 1$ and JAM-A (Kirchner et al., 2008)( PDB 3EOY) and should not preclude this interaction.

\subsection{Introduction of an epitope-encoding sequences before the stop codon in the S1 gene}

To insert tag-encoding sequence in the $\sigma 1$-encoding gene, the corresponding plasmid harbouring the type3 Dearing S1 gene under the control of the T7 polymerase was directly submitted to insertional site-directed mutagenesis, as described in Materials and methods. Infectious virus was recovered using transfection of BHK cells expressing the T7 RNA polymerase (Buchholz et al., 1999). These cells were used, as also described by others (Kobayashi et al., 2010), in replacement of a vaccinia virus vector expressing the T7 polymerase originally used for plasmid-based reovirus reverse genetics (Kobayashi et al., 2007). Viruses were recovered from single plaques and propagated, as described in Materials and methods.

The plaque assay procedure, modified to include chymotrypsin in the agar overlay was 
adapted to Vero cells. This facilitated the recovery of the plaques that were readily visualized on these cells by direct visual inspection or by phase contrast microscopy. However, the addition of chymotrypsin was necessary to obtain visible plaques (data not shown), consistent with previous report that proteolytic uncoating is a limiting step for reovirus infection in these cells (Golden et al., 2002). The Vero cells could eventually be an useful alternative to L929 cells to recover certain virus mutants since these cells are defective in interferon production (Desmyter et al., 1968; Emeny and Morgan, 1979).

As shown in figure 2, plaque size was comparable at either 2 or 4 days post-infection with either viruses. Only small plaques were observed at 48 hours for all viruses, although foci of infected cells could be detected by phase-contrast microscopy (data not shown). At 96 hours, plaques were readily visualized in all cases, even before staining. Final plaque size was apparently slightly increased for the HA-tagged virus, but the significance of this observation was not further examined.

\subsection{Sequencing the S1 gene of viral clones harbouring exogenous epitope}

The virus stocks obtained were then used to infect L929 cells and genomic RNA was extracted from viral particles. The nucleotide sequence of the gene was determined by RTPCR to ensure that the sequence encoding the tag was maintained without any other changes to the coding sequence. Six different clones of the hexahistidine-tagged virus were examined and 5 different clones of the HA-tagged virus. In each case, the sequence encoding the tag was retained by the virus. Except in one virus with the hexahistidine tag, harboring a glutamine to leucine amino acid substitution at position 51, there was no other change compared to the initial sequence of the cloned S1 gene (data not shown). 


\subsection{Expression of tagged $\sigma 1$ in infected cells}

The virus stocks encoding either wild-type or tagged $\sigma 1$ were then used to infect L929 cells and proteins were recovered for immunoblotting analysis. The two different tagged $\sigma 1$ appeared to be accumulated to similar levels as the wild-type protein under these conditions, as judged by immunoblotting using a polyclonal antibody specific to the globular head of the protein (Fig. 3A). When monoclonal antibodies specific to either the hexahistidine or HA tag were used (Fig. 3B and 3C, as indicated), the $\sigma 1$ protein was easily detected, only in cells infected with the respective virus; a small change in electrophoretic mobility was also noticed for the tagged proteins, as expected. Since there was no apparent secondary band of a mobility corresponding to the untagged protein, when the anti- $\sigma 1$ polyclonal antibody was used, it is most likely that the tag is stably retained on all copies of the protein and that $\sigma 1$ moieties incorporated to the viral particles are also harbouring the tag.

Immunoblotting of the same samples to detect the two major outer capsid proteins $\sigma 3$ and $\mu 1$ revealed similar amount of these proteins (Fig. 3D), with a similar ratio of the different $\sigma 1$ proteins compared to the other major outer capsid proteins.

\subsection{Addition of longer peptide sequences at the carboxyl-terminal end of $\sigma 1$}

Having demonstrated that it is possible to introduce exogenous genetic material encoding an exogenous peptide fused to the carboxyl-terminal end of $\sigma 1$, a plasmid construct was made that encodes the hexahistidine tag followed by a single SpeI site, in order to facilitate insertion of additional genetic material to determine the limitations in size and/or complexity that can be added at this position. A 96 nucleotide sequence, corresponding to three consecutive copies of the HA tag, separated by few amino acids (Fig. 1B) was recovered by PCR from an adequate plasmid and subcloned at the unique SpeI site, as described in Materials and Methods. Again, virus was easily recovered and forms plaques of similar size as 
the virus harbouring a single copy of HA (Fig 2). Immunoblotting analysis confirmed the presence of both the hexahistidine and HA-tag on the protein (Fig. 3B and 3C) and similar levels of the $\sigma 1, \sigma 3$ and $\mu 1$ proteins (Fig. 3A and 3D).

In order to determine if very long sequences can be successfully added at the same position, the coding sequence for the enhanced yellow fluorescent protein (239 amino acids) was amplified by PCR from a plasmid construct and introduced at the SpeI site to make a fusion protein with $\sigma 1$ (Fig. 1A). To verify if the efficiency of virus recovery will be affected if only the nucleotide sequence is present, without actual fusion at the protein level, this construct was then subjected to site-directed mutagenesis to introduce a premature stop codon 3 amino acids after the beginning of the YFP-encoding sequence (Fig. 1A). However, despite numerous attempts, it was not possible to recover viruses with either of these two plasmid constructs.

\subsection{Further characterization of tagged viruses}

In order to further establish the replicative potential of the tagged viruses, the kinetic of production of infectious virus following infection of L929 cells at a low multiplicity of infection was measured by viral titration (Fig. 4). All three tagged viruses behave similarly and were somewhat delayed compared to wild-type virus, although final titers were only reduced less than tenfold (Fig. 4 and data not shown). When virus binding at the cell surface was compared using a newly developed FACS-based assay, binding was reduced for all three tagged viruses, either as virions or ISVPs, compared to the wild type virus (data not shown). It was not established if this is due to reduced incorporation of tagged $\sigma 1$ to the virions or reduced affinity of the $\sigma 1$ for its cellular receptors due to partial interference by the tag. 


\section{Discussion}

Before the present work was undertaken, is has been reported that a small hexahistidine tag could be added to the carboxyl-terminal end of $\sigma 1$ (van den Wollenberg et al., 2008). In this previous study, "recombinant" viruses were selected for their ability to bind and replicate solely in cells engineered to express an artificial cell surface receptor made of a single-chain antibody against the tag. The mechanism allowing the recovery of the tagged encoding sequence in a viral RNA with authentic 5' and 3' untranslated regions remains unknown. In these viruses, additional amino acids changes were however "selected" in the tagged $\sigma 1$ protein compared to the original protein but this was not observed in the present study using the more direct plasmid-based reverse genetics approach. The only other amino acid substitution obtained was found in only one randomly-picked plaques and close to the aminoterminal end, and thus far from the carboxy-terminal tag position. The requirement for a coinfecting virus in the previous approach suggest that the virus stock used contained viruses whose sequence differs from the consensus and this is later reflected in the sequence of viruses from randomly picked plaques. Clearly, when direct plasmid-based reverse genetics is used to incorporate the exogenous sequence, there is no requirement for such "second-site" mutations; furthermore, the reverse genetics approach is obviously more direct and does not require a special cell line for selecting the novel viruses.

The carboxyl-terminal end of $\sigma 1$ thus appears ideally suited to pursue the idea of adding additional peptide at the surface of the reovirus particles. The question is not trivial since other attempts to add exogenous epitopes on other viral proteins had little success (Rouault and Lemay, 2003; Lemay, 2011; and unpublished data). The limits in length that could be accepted at the carboxyl-terminal end of $\sigma 1$, while maintaining the infectious potential of the viral particle, remains to be determined; the longest tagged $\sigma 1$ with its triple HA tag has a total of 40 additional amino acids (Fig. 1A). The construct encoding the 
hexahistidine epitope followed by a unique $S p e I$ restriction will facilitate further work to determine the limitations in size that can be added without affecting viral replication too stongly; the presence of the hexahistidine epitope could play the role of a linker to facilitate insertion of additional amino acids at this position. The addition of even the smallest tag does affect virus binding to a certain extent, although this remains acceptable for most applications; however, it may prove difficult to add much longer and complex peptides without further, and more drastic, consequences on virus infectivity.

In addition to possible consequences of amino acids addition on the protein itself, the importance of nucleotide sequence and structure at both termini are likely of importance in allowing transcription, replication and encapsidation of RNA genomic segments.

In conclusion, additional genetic material could be added to the genome of a replication-competent reovirus using plasmid-based reverse genetics, and up to at least 120 nucleotides can be added before the authentic 3' untranslated region of S1. In addition, the ability to modulate reovirus' ability to replicate in different cell types, by addition of specific microRNA targets, could thus be also envisaged for reovirus. This could add to the specificity of viral infection toward specific cell types and could make safer viruses for use as virotherapy agents, as currently investigated for other oncolytic viruses (reviewed in (Bell and Kirn, 2008; Kelly et al., 2008)). The plasmid-based reverse genetics for the addition of exogenous nucleic acids sequence to the viral genome and/or peptide sequences to viral proteins thus opens novel fields of investigation and applications for mammalian reoviruses as virotherapy agents. 


\section{Acknowledgments}

This work was supported by a NSERC discovery grant to G.L. V.B.-L. was the recipient of a studentship from the "Fondation Gabriel Marquis" of Université de Montréal. We thank Véronique Sandekian for her help with some experiments and for numerous discussions.

\section{References}

Alain, T., Kim, T.S., Lun, X., Liacini, A., Schiff, L.A., Senger, D.L., Forsyth, P.A., 2007. Proteolytic disassembly is a critical determinant for reovirus oncolysis. Mol. Ther. 15, $1512-1521$.

Antar, A.A., Konopka, J.L., Campbell, J.A., Henry, R.A., Perdigoto, A.L., Carter, B.D., Pozzi, A., Abel, T.W., Dermody, T.S., 2009. Junctional adhesion molecule-A is required for hematogenous dissemination of reovirus. Cell Host Microbe 5, 59-71.

Bell, J.C., Kirn, D., 2008. MicroRNAs fine-tune oncolytic viruses. Nat. Biotechnol. 26, 13461348.

Blechacz, B., Russell, S.J., 2008. Measles virus as an oncolytic vector platform. Curr. Gene Ther. 8, 162-175.

Boehme, K.W., Guglielmi, K.M., Dermody, T.S., 2009. Reovirus nonstructural protein sigma1s is required for establishment of viremia and systemic dissemination. Proc. Natl. Acad. Sci. U. S. A. 106, 19986-19991.

Buchholz, U.J., Finke, S., Conzelmann, K.K., 1999. Generation of bovine respiratory syncytial virus (BRSV) from cDNA: BRSV NS2 is not essential for virus replication in tissue culture, and the human RSV leader region acts as a functional BRSV genome promoter. J. Virol. 73, 251-259.

Campadelli-Fiume, G., De Giovanni, C., Gatta, V., Nanni, P., Lollini, P.-L., Menotti, L., 2011. 
Rethinking herpes simplex virus: the way to oncolytic agents. Rev. Med. Virol. May 27. doi: 10.1002/rmv.691. [Epub ahead of print]

Chappell, J.D., Prota, A.E., Dermody, T.S., Stehle, T., 2002. Crystal structure of reovirus attachment protein sigma1 reveals evolutionary relationship to adenovirus fiber. EMBO J. 21, 1-11.

Coyne, C.B., 2009. The distinct roles of JAM-A in reovirus pathogenesis. Cell Host Microbe $5,3-5$.

Danthi, P., Coffey, C.M., Parker, J.S., Abel, T.W., Dermody, T.S., 2008a. Independent regulation of reovirus membrane penetration and apoptosis by the mul phi domain. PLoS Pathog. 4, e1000248.

Danthi, P., Kobayashi, T., Holm, G.H., Hansberger, M.W., Abel, T.W., Dermody, T.S., 2008b. Reovirus apoptosis and virulence are regulated by host cell membrane penetration efficiency. J. Virol. 82, 161-172.

Danthi, P., Guglielmi, K.M., Kirchner, E., Mainou, B., Stehle, T., Dermody, T.S., 2010. From touchdown to transcription: the reovirus cell entry pathway. Curr. Top. Microbiol. Immunol. 343, 91-119.

Desmyter, J., Melnick, J.L., Rawls, W.E., 1968. Defectiveness of interferon production and of rubella virus interference in a line of African green monkey kidney cells (Vero). J. Virol. 2, 955-961.

Emeny, J.M., Morgan, M.J., 1979. Regulation of the interferon system: evidence that Vero cells have a genetic defect in interferon production. J. Gen. Virol. 43, 247-252.

Forrest, J.C., Dermody, T.S., 2003. Reovirus receptors and pathogenesis. J. Virol. 77, 91099115.

Golden, J.W., Linke, J., Schmechel, S., Thoemke, K., Schiff, L.A., 2002. Addition of exogenous protease facilitates reovirus infection in many restrictive cells. J. Virol. 76, 
7430-7443.

Harrington, K.J., Vile, R.G., Melcher, A., Chester, J., Pandha, H.S., 2010. Clinical trials with oncolytic reovirus: Moving beyond phase I into combinations with standard therapeutics. Cytokine Growth Factor Rev. 21, 91-98.

Kelly, E.J., Hadac, E.M., Greiner, S., Russell, S.J., 2008. Engineering microRNA responsiveness to decrease virus pathogenicity. Nat. Med. 14, 1278-1283.

Kelly, K., Nawrocki, S., Mita, A., Coffey, M., Giles, F.J., Mita, M., 2009. Reovirus-based therapy for cancer. Expert Opin. Biol. Ther. 9, 817-830.

Kim, M., Garant, K.A., zur Nieden, N.I., Alain, T., Loken, S.D., Urbanski, S.J., Forsyth, P.A., Rancourt, D.E., Lee, P.W.K., Johnston, R.N., 2011. Attenuated reovirus displays oncolysis with reduced host toxicity. Br. J. Cancer 104, 290-299.

Kirchner, E., Guglielmi, K.M., Strauss, H.M., Dermody, T.S., Stehle, T., 2008. Structure of reovirus sigma1 in complex with its receptor junctional adhesion molecule-A. PLoS Pathog. 4, e1000235.

Kobayashi, T., Antar, A.A., Boehme, K.W., Danthi, P., Eby, E.A., Guglielmi, K.M., Holm, G.H., Johnson, E.M., Maginnis, M.S., Naik, S., Skelton, W.B., Wetzel, J.D., Wilson, G.J., Chappell, J.D., Dermody, T.S., 2007. A plasmid-based reverse genetics system for animal double-stranded RNA viruses. Cell Host Microbe 1, 147-157.

Kobayashi, T., Ooms, L.S., Chappell, J.D., Dermody, T.S., 2009. Identification of functional domains in reovirus replication proteins muNS and mu2. J. Virol. 83, 2892-2906.

Kobayashi, T., Ooms, L.S., Ikizler, M., Chappell, J.D., Dermody, T.S., 2010. An improved reverse genetics system for mammalian orthoreoviruses. Virology 398, 194-200.

Kolodziej, P.A., Young, R.A., 1991. Epitope tagging and protein surveillance. Meth. Enzymol. $194,508-519$. 
Lal, R., Harris, D., Postel-Vinay, S., de Bono, J., 2009. Reovirus: Rationale and clinical trial update. Curr. Opin. Mol. Ther. 11, 532-539.

Lemay, G., 2011. La génétique inverse dans l'étude des réovirus: Progrès, obstacles et développements futurs. Virologie 15, 53-62.

Loken, S.D., Norman, K., Hirasawa, K., Nodwell, M., Lester, W.M., Demetrick, D.J., 2004. Morbidity in immunosuppressed (SCID/NOD) mice treated with reovirus (Dearing 3) as an anti-cancer biotherapeutic. Cancer Biol. Ther. 3, 734-738.

Marcato, P., Shmulevitz, M., Pan, D., Stoltz, D., Lee, P.W., 2007. Ras transformation mediates reovirus oncolysis by enhancing virus uncoating, particle infectivity, and apoptosisdependent release. Mol. Ther. 15, 1522-1530.

Mathis, J.M., Stoff-Khalili, M.A., Curiel, D.T., 2005. Oncolytic adenoviruses - selective retargeting to tumor cells. Oncogene $24,7775-7791$.

Mercier, G.T., Campbell, J.A., Chappell, J.D., Stehle, T., Dermody, T.S., Barry, M.A., 2004. A chimeric adenovirus vector encoding reovirus attachment protein sigma1 targets cells expressing junctional adhesion molecule 1. Proc. Natl. Acad. Sci. U. S. A. 101, 61886193.

Middleton, J.K., Agosto, M.A., Severson, T.F., Yin, J., Nibert, M.L., 2007. Thermostabilizing mutations in reovirus outer-capsid protein $\mu 1$ selected by heat inactivation of infectious subvirion particles. Virology 361, 412-425.

Parashar, K., Tarlow, M.J., McCrae, M.A., 1992. Experimental reovirus type 3-induced murine biliary tract disease. J. Pediatr. Surg. 27, 843-847.

Rouault, É., Lemay, G. 2003. Incorporation of epitope-tagged viral $\sigma 3$ proteins to reovirus virions. Can. J. Microbiol. 49, 407-417.

Rudd, P. and Lemay, G., 2005. Correlation between interferon sensitivity of reovirus isolates 
and ability to discriminate between normal and Ras-transformed cells. J. Gen. Virol. 86, 1489-97.

Schagen, F.H., Wensveen, F.M., Carette, J.E., Dermody, T.S., Gerritsen, W.R., van Beusechem, V.W., 2006. Genetic targeting of adenovirus vectors using a reovirus sigma1-based attachment protein. Mol. Ther. 13, 997-1005.

Schelling, P., Campbell, J.A., Stehle, T., Dermody, T.S. 2008. Structural basis of mammalian orthoreovirus cell attachment. In: Patton, J.T. (Ed), Segmented double-stranded RNA viruses: structure and molecular biology, Caister Academic Press, pp. 147-171.

Schneider, B.L., Seufert, W., Steiner, B., Yang, Q.H., Futcher, A.B., 1995. Use of polymerase chain reaction epitope tagging for protein tagging in Saccharomyces cerevisiae. Yeast $11,1265-1274$

Shmulevitz, M., Pan, L.Z., Garant, K., Pan, D., Lee, P.W., 2010. Oncogenic Ras promotes reovirus spread by suppressing IFN-beta production through negative regulation of RIG-I signaling. Cancer Res. 70, 4912-4921.

Smakman, N., van den Wollenberg, D.J., Borel Rinkes, I.H., Hoeben, R.C., Kranenburg, O., 2005. Sensitization to apoptosis underlies KrasD12-dependent oncolysis of murine C26 colorectal carcinoma cells by reovirus T3D. J. Virol. 79, 14981-14985.

Strong, J.E., Coffey, M.C., Tang, D., Sabinin, P., Lee, P.W., 1998. The molecular basis of viral oncolysis: usurpation of the Ras signaling pathway by reovirus. EMBO J. 17, 33513362.

Thirukkumaran, C., Morris, D.G., 2009. Oncolytic viral therapy using reovirus. Methods Mol. Biol. 542, 607-634.

Tsuruta, Y., Pereboeva, L., Glasgow, J.N., Luongo, C.L., Komarova, S., Kawakami, Y., Curiel, D.T., 2005. Reovirus fiber incorporated into adenovirus serotype 5 enhances infectivity via a CAR-independent pathway. Biochem. Biophys. Res. Commun. 335, 
205-214.

Tsuruta, Y., Pereboeva, L., Glasgow, J.N., Rein, D.T., Kawakami, Y., Alvarez, R.D., Rocconi, R.P., Siegal, G.P., Dent, P., Fisher, P.B., Curiel, D.T., 2007. A mosaic fiber adenovirus serotype 5 vector containing reovirus sigma 1 and adenovirus serotype 3 knob fibers increases transduction in an ovarian cancer ex vivo system via a coxsackie and adenovirus receptor-independent pathway. Clin. Cancer Res. 13, 2777-2783.

Tyler, K.L., Sokol, R.J., Oberhaus, S.M., Le, M., Karrer, F.M., Narkewicz, M.R., Tyson, R.W., Murphy, J.R., Low, R., Brown, W.R., 1998. Detection of reovirus RNA in hepatobiliary tissues from patients with extrahepatic biliary atresia and choledochal cysts. Hepatology 27, 1475-1482.

van den Wollenberg, D.J., van den Hengel, S.K., Dautzenberg, I.J., Cramer, S.J., Kranenburg, O., Hoeben, R.C., 2008. A strategy for genetic modification of the spike-encoding segment of human reovirus T3D for reovirus targeting. Gene Ther. 15, 1567-1578.

Van Den Wollenberg, D.J., Van Den Hengel, S.K., Dautzenberg, I.J., Kranenburg, O., Hoeben, R.C., 2009. Modification of mammalian reoviruses for use as oncolytic agents. Expert Opin. Biol. Ther. 9, 1509-1520.

van Houdt, W.J., Smakman, N., van den Wollenberg, D.J., Emmink, B.L., Veenendaal, L.M., van Diest, P.J., Hoeben, R.C., Borel Rinkes, I.H., Kranenburg, O., 2008. Transient infection of freshly isolated human colorectal tumor cells by reovirus T3D intermediate subviral particles. Cancer Gene Ther. 15, 284-292.

Virgin, H.W., Mann, M.A., Fields, B.N., Tyler, K.L., 1991. Monoclonal antibodies to reovirus reveal structure/function relationships between capsid proteins and genetics of susceptibility to antibody action. J. Virol. 65, 6772-6781.

Wilson, I.A., Niman, H.L., Houghten, R.A., Cherenson, A.R., Connolly, M.L., Lerner, R.A., 
1984. The structure of an antigenic determinant in a protein. Cell 37, 767-778.

Zurney, J., Kobayashi, T., Holm, G.H., Dermody, T.S., Sherry, B., 2009. Reovirus mu2 protein inhibits interferon signaling through a novel mechanism involving nuclear accumulation of interferon regulatory factor 9. J. Virol. 83, 2178-2187. 


\section{Figures legends}

Fig. 1. Sequence and structure at the carboxyl-terminal end of tagged $\sigma 1$ proteins. (A) Amino acids sequence at the carboxyl-terminal end of either the wild-type or the different tagged version of $\sigma 1$ used in this manuscript. (B) Crystallographic structure (from PDB 1KKE) of the $\sigma 1$ homotrimer viewed from the side with the arrow pointing at the position of the carboxylterminal amino acid illustrated as a sphere. (C) Crystallographic structure (from PDB 3EOY) of the complex between the $\sigma 1$ homotrimer and the JAM-A cellular receptor viewed from the top of the structure with the arrow pointing at the position of the carboxyl-terminal amino acid illustrated as a sphere.

Fig.2. Plaque formation by the tagged viruses. Plaque assay was performed on Vero cells in the presence of chymotrypsin, as described in Materials and methods. A single well presenting well separated plaques are presented for the wild-type (wt), the hexahistidine-tagged (Reo.S1.6H), the HA-tagged (Reo.S1.HA) and the triple-HA tagged (Reo.S1.3HA) viruses. Cell monolayers were fixed and stained at either 48 or 96 hours post-infection, as described in Materials and Methods.

Fig. 3. Immunoblotting of viral proteins in cells infected with the tagged viral particles. Viral stocks of wild-type, $6 \mathrm{H}$-tagged or HA-tagged were prepared and titrated as described in Materials and methods. Murine L929 cells were infected with these viruses at a multiplicity of infection of 2 PFU/cell. Proteins were recovered from infected cells 24 hours later and equivalent amounts were loaded on SDS-PAGE and analyzed by immunoblotting. (A) The polyclonal monospecific anti- $\sigma 1$ head antibody, the monoclonal anti-HA antibody (B), or the monoclonal anti-hexahistidine antibody (C) were used, as indicated. (D) The membrane 
previously probed with the polyclonal monospecific anti- $\sigma 1$ head antibody was reprobed with monoclonal anti- $\mu 1$ antibody. (E) The membrane previously probed with the anti-HA antibody was reprobed with the anti-tubulin antibody.

Fig.4. Replication of tagged viruses. Wild-type or tagged viruses were used to infect L929 cells at a MOI of approximately 0.5 (upper panel) and 0.05 (lower panel) PFU/cell. Petri dishes of infected cells were recovered at 12, 24 or 48 hours post-infection and virus titration was performed by plaque assay, as described in Materials and Methods. 


\section{Table I}

\begin{tabular}{|c|c|c|c|}
\hline Use & virus & Primer & Primer sequence* \\
\hline Mutagenesis & REO.S1.6H & $\begin{array}{l}\text { S1-6HIS } \\
\text { S1-6HIS-AS }\end{array}$ & $\begin{array}{l}\text { GCGTAGTTTCACGCATCACCATCACCATCACTGAGGATCAGACCAC } \\
\text { GTGGTCTGATCCTCAGTGATGGTGATGGTGATGCGTGAAACTACGC }\end{array}$ \\
\hline Mutagenesis & Not determined & $\begin{array}{l}\text { S1-SpeI } \\
\text { S1-SpeI-AS }\end{array}$ & $\begin{array}{l}\text { GCGTAGTTTCACGCATCACCATCACCATCACACTAGTTGAGGATCAGACCAC } \\
\text { GTGGTCTGATCCTCAACTAGTGTGATGGTGATGGTGATGCGTGAAACTACGC }\end{array}$ \\
\hline Mutagenesis & Reo.S1.HA & $\begin{array}{l}\mathrm{S} 1-\mathrm{HA} \\
\mathrm{S} 1-\mathrm{HA}-\mathrm{AS}\end{array}$ & $\begin{array}{l}\text { GCGTAGTTTCACGTACCCATACGACGTCCCAGACTACGCTTGAGGATCAGACCAC } \\
\text { GTGGTCTGATCCTCAAGCGTAGTCTGGGACGTCGTATGGGTACGTGAAACTACGC }\end{array}$ \\
\hline Mutagenesis & Negative & $\begin{array}{l}\text { S1-YFP-STOP } \\
\text { S1-YFP-STOP-AS }\end{array}$ & $\begin{array}{l}\text { CACACTAGTATGGTGAGCTAGGGCGAGGAGCTGTTC } \\
\text { GAACAGCTCCTCGCCCTAGCTCACCATACTAGTGTG }\end{array}$ \\
\hline $\operatorname{PCR}$ (3HA) & Reo.S1.3HA & $\begin{array}{l}\mathrm{S} 1-3 \mathrm{HA}-5^{\prime} \\
\mathrm{S} 1-3 \mathrm{HA}-3^{\prime}\end{array}$ & $\begin{array}{l}\text { CGGCCGCACTAGTTACCCATACG } \\
\text { GGCCGCAACTAGTAGCGTAATCTGG }\end{array}$ \\
\hline PCR (YFP) & Negative & $\begin{array}{l}\text { YFP-Spe-5' } \\
\text { YFP-Spe-3' }\end{array}$ & $\begin{array}{l}\text { GCGCCGACTAGTATGGTGAGCAAGGGCGAGCTGTTC } \\
\text { CAGGACACTAGTTTACTTGTACAGCTCGTCCATGCCGAG }\end{array}$ \\
\hline RT-PCR & & $\begin{array}{l}\mathrm{S} 1(1-15) \\
\mathrm{S} 1(1416-1394)\end{array}$ & $\begin{array}{l}\text { GCTATTGGTCGGATG } \\
\text { AGTGCCGCGGGGTGGTCTGATC }\end{array}$ \\
\hline \multicolumn{2}{|c|}{ Sequencing $\mathrm{S} 1(1-15)$} & $\begin{array}{l}\mathrm{S} 1(1-15) \\
\mathrm{S} 1(708-730) \\
\mathrm{S} 1(917-940)\end{array}$ & $\begin{array}{l}\text { GCTATTGGTCGGATG } \\
\text { GACTCTCAAGACGACTGTGTTTG } \\
\text { ATGTTAGCGGCGGTATCGGAATGA }\end{array}$ \\
\hline
\end{tabular}

*The SpeI site is indicated in red; the sequence encoding the $6 \mathrm{His} \mathrm{tag}$ is indicated in green; sequences in blue are complementary to YFP-encoding sequence while purple sequences are complementary to sequences encoding the HA epitope. 


\section{Figure 1}
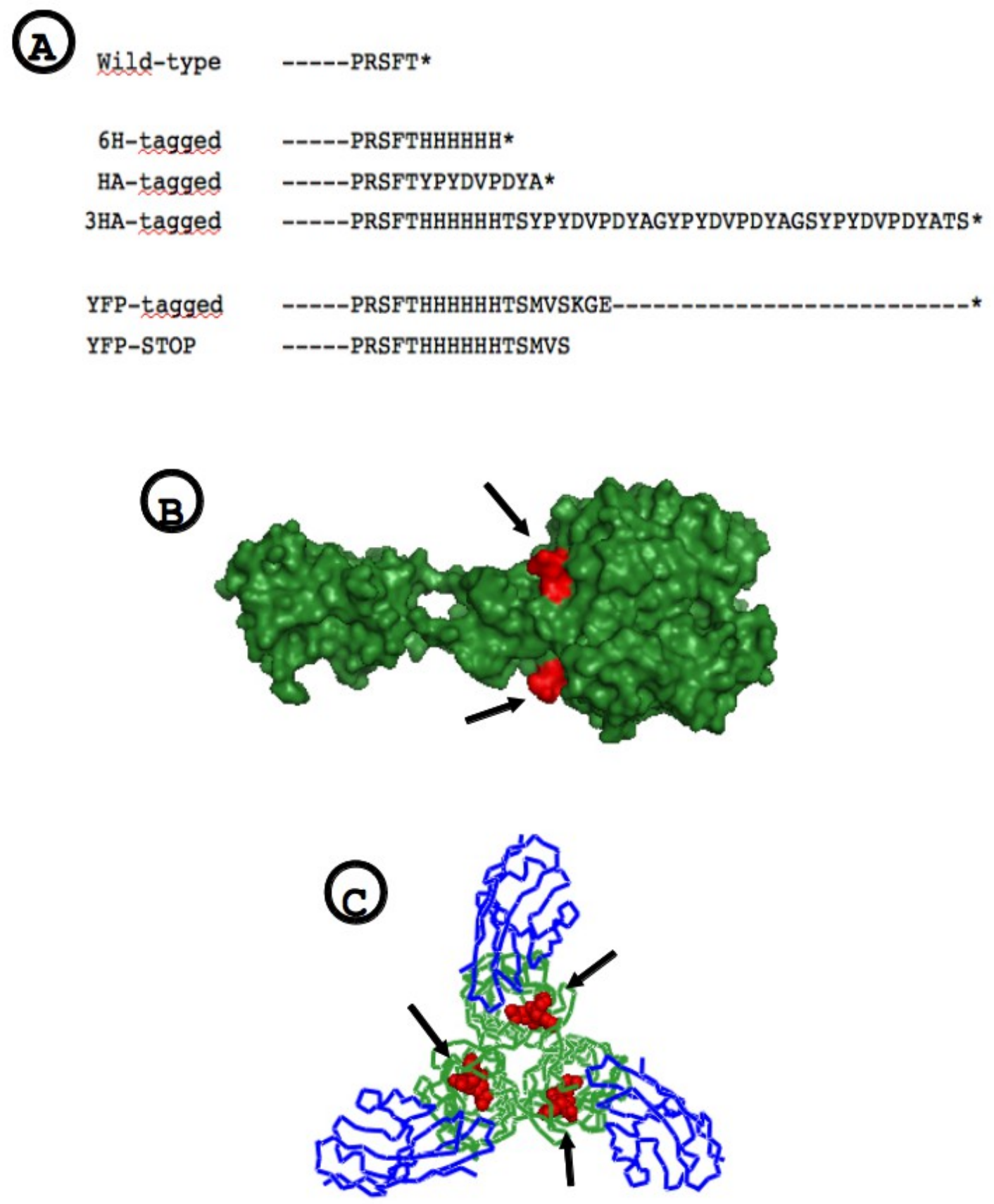


\section{Figure 2}

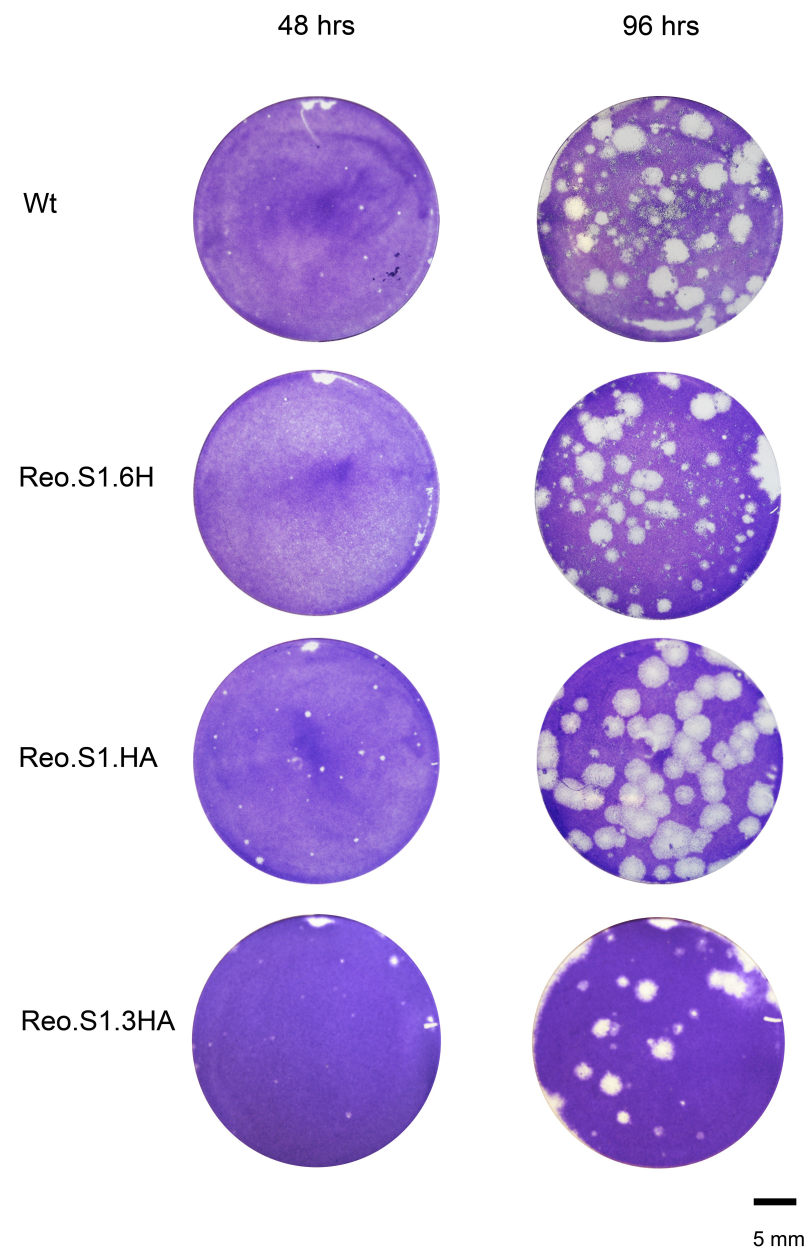




\section{Figure 3}

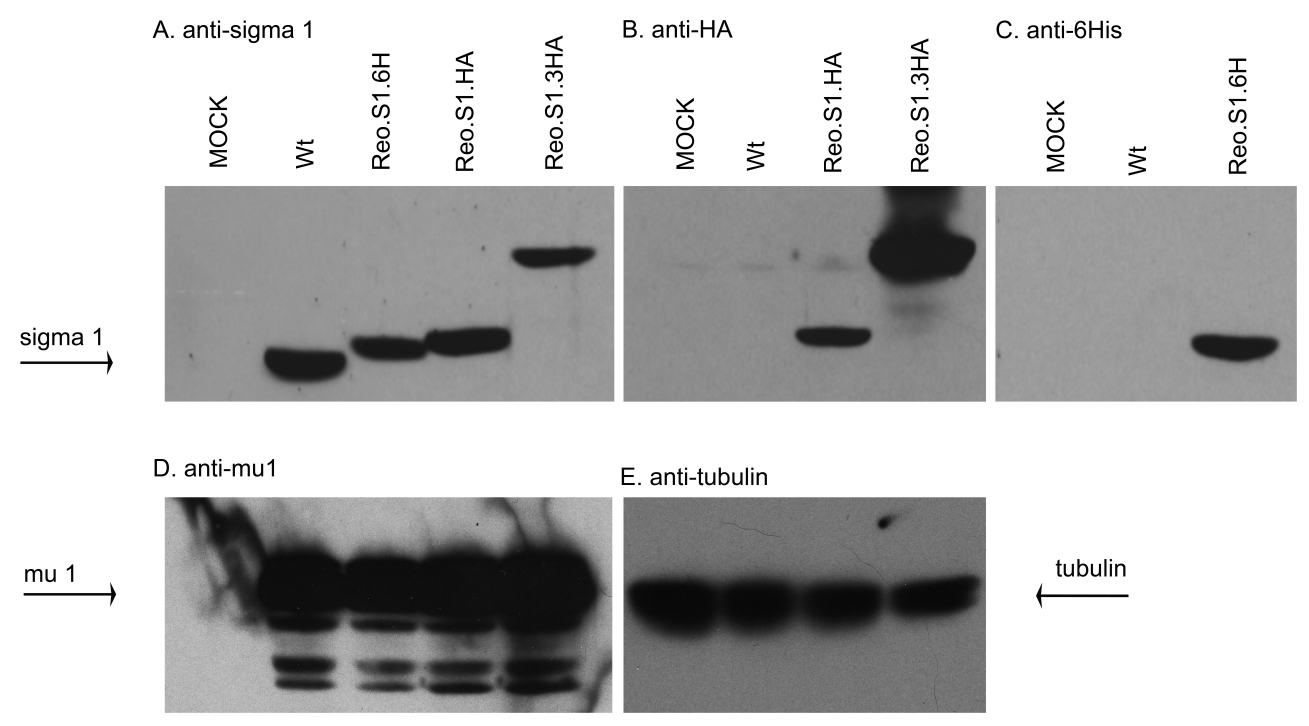


Figure 4
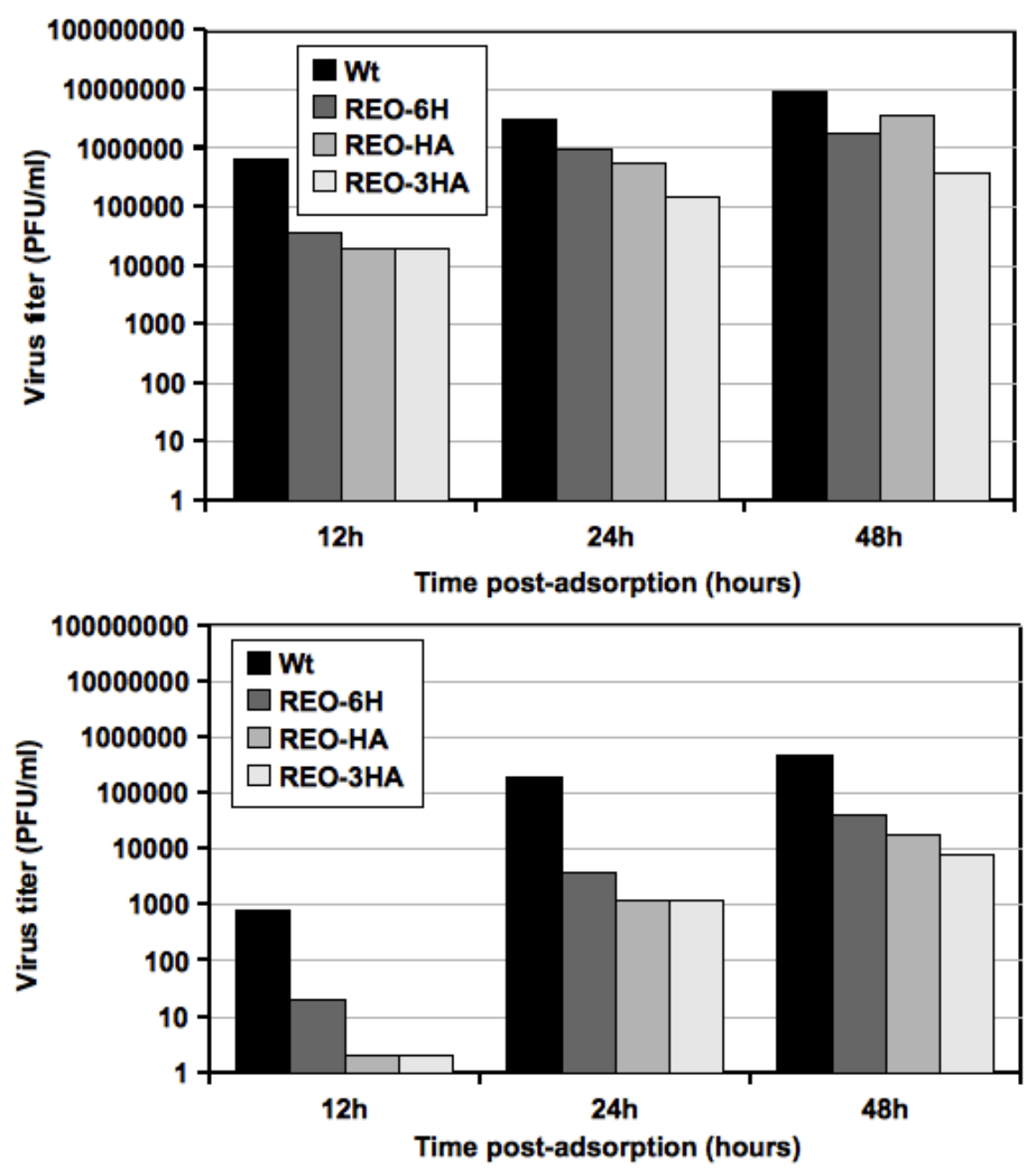